\title{
Hygienic Source-Code Generation Using Functors
}

\author{
Karl Crary \\ Carnegie Mellon University
}

\begin{abstract}
Existing source-code-generating tools such as Lex and Yacc suffer from practical inconveniences because they use disembodied code to implement actions. To prevent this problem, such tools could generate closed functors that are then instantiated by the programmer with appropriate action code. This results in all code being type checked in its appropriate context, and it assists the type checker in localizing errors correctly. We have implemented a lexer generator and parser generator based on this technique for Standard ML, OCaml, and Haskell.
\end{abstract}

\section{Introduction}

Compiler implementers have a love-hate relationship with source-code-generating tools such as Lex 9. (which generates lexers from regular expressions) and Yacc [7] (which generates shift-reduce parsers from context-free grammars). These tools automate the some of the most tedious parts of implementing a parser, but they can be awkward to use.

One of the main awkward aspects of such tools is the disembodied code problem. To build a lexer or a parser, these tools cobble together snippets of code (each implementing an action of the lexer/parser) supplied by the programmer in a lexer/parser specification file. Unfortunately, the code snippets, as they appear in the specification file, are divorced from their ultimate context. The tools manipulate them as simple strings $\square$

This makes programming awkward in several ways. Functions and other values are passed into the snippets using identifiers that are bound nowhere in the programmer's code, nor even introduced by a pseudo-binding such as open. Rather, the snippet is copied into a context in which such identifiers are in scope. This can make code difficult to read.

More importantly, disembodied code makes debugging challenging, because the code seen by the compiler bears little resemblance to the code written by the programmer. For example, consider the following line from an ML-Lex [1] specification:

$$
\{\text { whitespace }\}+=>(\operatorname{lex}()) \text {; }
$$

This line tells the lexer to skip any whitespace it encounters by matching it and then calling itself recursively to continue.

\footnotetext{
${ }^{1}$ Such strings may even include syntax errors, which are duly copied into the output code. Typically the tool does not even ensure that delimiters are matched.
}

(Note that lex is an example of an identifier introduced implicitly when the snippet is copied.) ML-ULex ${ }^{2}[13$ converts the line into the Standard ML code:

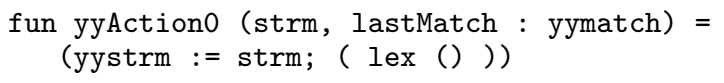

This output code already is not very easy to read. However, the problem is greatly exacerbated by the familiar phenomenon in typed functional languages that type checkers are often bad at identifying the true source of a type error. Suppose we introduce an error into the specification by omitting the argument to lex:

$$
\{\text { whitespace }\}+\Rightarrow(\text { lex }) \text {; }
$$
like:

We now obtain ${ }^{3}$ several pages of error messages looking

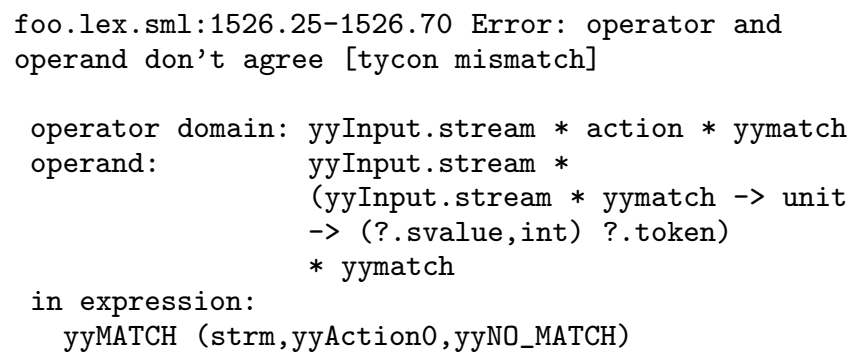

or like:

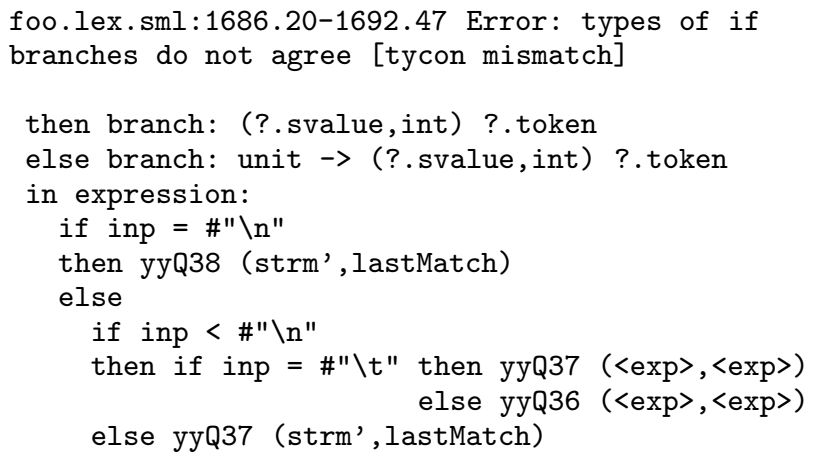

${ }^{2}$ The lexer generator (compatible with ML-Lex) that Standard ML of New Jersey uses.

${ }^{3}$ Using Standard ML of New Jersey v100.68. 
and none of the errors is anywhere near the copied snippet containing the error.

The problem is related to the issue of variable hygiene in macro expansion 8. In both cases, the programmer writes code (a lexer/parser action, or macro argument) divorced from its ultimate context and then - after processing - that code is dropped verbatim into its ultimate context. In the setting of macros, this sets the scene for variable capture to occur, which is nearly always erroneous. In lexer generators, variable capture often is actually desired (consider the lex identifier), but, as observed above, it is nevertheless difficult to reason about and to debug.

Accordingly, we are interested in source-code generation in which all code is type-checked in the same context in which it is written. We call this hygienic source-code generation by analogy to hygienic macro expansion, which ensures the same thing for macros.

An obvious way to accomplish hygienic source-code generation is to have the tool type-check every snippet before it assembles them into output code. But, this approach is unattractive in practice, because it necessitates including all the apparatus of parsing, elaboration, and type-checking as part of a tool that does not otherwise need all that apparatus.

We propose a simpler and cleaner alternative: Rather than type-check disembodied code in context, we dispense with disembodied code altogether. To accomplish this, the tool-rather than assembling snippets of source code into a program - generates a functor that abstracts over the code that used to reside in snippets. The programmer then applies the functor in order to instantiate the lexer or parser with specific action implementations.

A third alternative, arguably more principled than ours, is to implement the lexer/parser generator in a type-safe metaprogramming language such as MetaML 12 or its cousins. With such an approach, as in ours, the action implementations would be type-checked in context, without any need to duplicate compiler apparatus. Furthermore, it would remove the need to write the lexer/parser specification and action implementations in two separate places, as our proposal requires. On the other hand, this alternative requires one to use a special programming language. We want an approach compatible with pre-existing, conventional functional programming languages, specifically $\mathrm{ML}$ and Haskell.

Finally, in some problem domains one may consider avoiding generated source code entirely. For example, in parsing, some programmers find parser combinators [5, 6] to be a suitable or even preferable alternative to Yacc-like tools. Nevertheless, many programmers prefer traditional LR parser generators for various reasons including error reporting and recovery, and ambiguity diagnostics. In this work we take it as given that source-code generation is preferred, for whatever reason.

At first blush, our proposal might seem to replace one sort of disembodied code with another. This is true in a sense, but there is a key difference. The code in which the functor is applied is ordinary code, submitted to an ordinary compiler. That compiler then type checks the action code (that formerly resided in snippets) in the context in which it now appears, which is the functor's argument.

As a practical matter, each action becomes a distinct field of the functor argument, and consequently each action is type-checked independently, as desired. The type of the functor is already known, so an error in one action will not be misinterpreted as an error in all the other actions.

Employing this design, we have implemented a lexer generator, called CM-Lex, and a parser generator, called CM-Yacc. Each tool supports Standard ML, OCaml, and Haskell 4 Both tools are available on-line at:

$$
\text { www.cs.cmu.edu/ crary/cmtool/ }
$$

In the remainder of the paper we describe how the tools work, taking the lexer generator as our primary example.

\section{Lexing Functor Generation}

The following is a very simple CM-Lex specification:

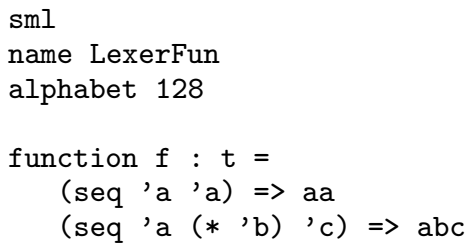

The specification's first line indicates that CM-Lex should generate Standard ML code. The next two lines indicate that CM-Lex should produce a functor named LexerFun, and that it should generate a 7-bit parser (any symbols outside the range $0 \ldots 127$ will be rejected automatically).

The remainder gives the specification of a lexing function named $f$. The function will return a value of type $t$, and it is defined by two regular expressions. Regular expressions are given as S-expressions using the Scheme Shell's SRE notation [1].

Thus, the first arm activates an action named aa when the regular expression $a a$ is recognized. The second activates an action named abc when the regular expression $a b^{*} c$ is recognized.

Observe that the specification contains no disembodied code. The actions are simply given names, which are instantiated when the resulting functor is applied.

From this specification, CM-Lex generates the following Standard ML code 6

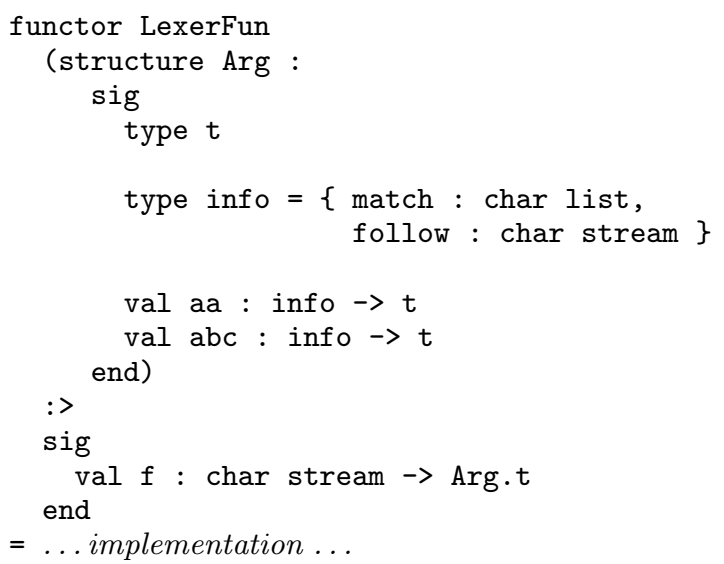

\footnotetext{
${ }^{4}$ The tool is implemented in Standard ML.

${ }^{5}$ Although SREs are less compact than some other notations, we find their syntax is much easier to remember.

${ }^{6}$ We simplify here and in the following examples for the sake of exposition.
} 


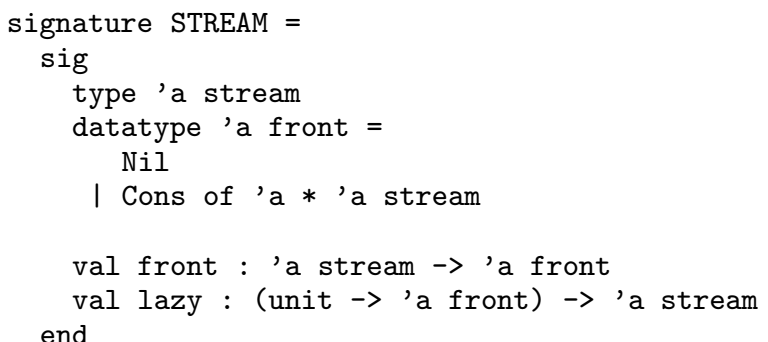

Figure 1: Lazy Streams

When the programmer calls the functor, he provides the type $t$ and the actions aa and abc, both of which produce $a t$ from a record of matching information. The functor then returns a lexing function $f$, which produces a $t$ from a stream of characters.

Although the programmer-supplied actions can have side effects, the lexer itself is purely functional. The input is processed using lazy streams (the signature for which appears in Figure 1). Each action is given the portion of the stream that follows the matched string as part of the matching information.

As an illustration of how the functor might be applied, the following program processes an input stream, printing a message each time it recognizes a string:

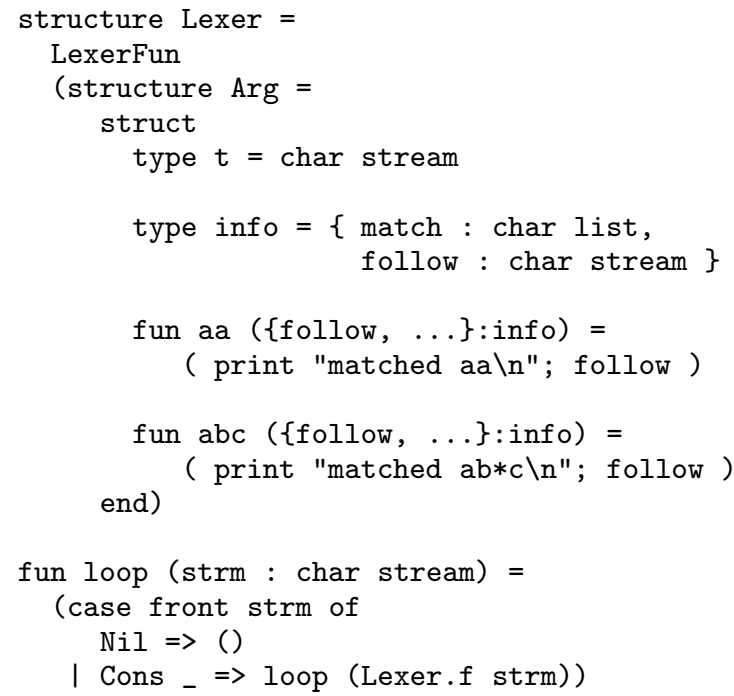

The function Lexer.f matches its argument against the two regular expressions and calls the indicated action, each of which prints a message and returns the remainder of the stream.

Observe that the implementations of the actions (the fields aa and $a b c$ of the argument structure) are ordinary ML code. As one consequence, the action code faces the standard type checker. Moreover, each action's required type is unambiguously given by LexerFun's signature and the type argument $t$, so error identification is much more accurate.

For example, suppose we replace the aa action with an erroneous implementation that fails to return the remainder of the stream:

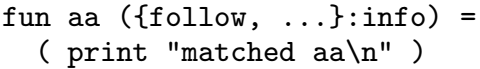

The type checker is able to identify the source of the error precisely, finding that aa has the type unit instead of $t$ :

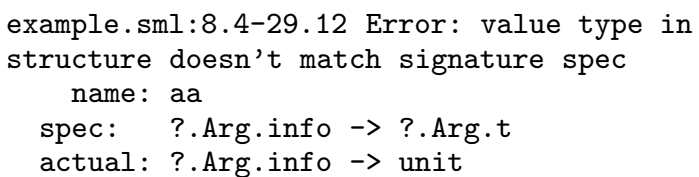

\subsection{An expanded specification}

We may add a second function to the lexer by simply adding another function specification:

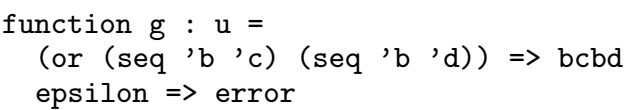

In the parlance of existing lexer generators, multiple functions are typically referred to as multiple start conditions or start states, but we find it easier to think about them as distinct functions that might or might not share some actions. In this case, the function $g$ is specified to return a value of type $u$. Since $u$ might not be the same type as $t, g$ cannot share any actions with $f$.

The first arm activates an action named bcbd when the regular expression $b c+b d$ is recognized. The second arm activates an action named error when the empty string is recognized. Like other lexer generators, CM-Lex prefers the longest possible match, so an epsilon arm will only be used when the input string fails to match any other arm. Thus, the latter arm serves as an error handler 7

From the expanded specification, CM-Lex generates the functor:

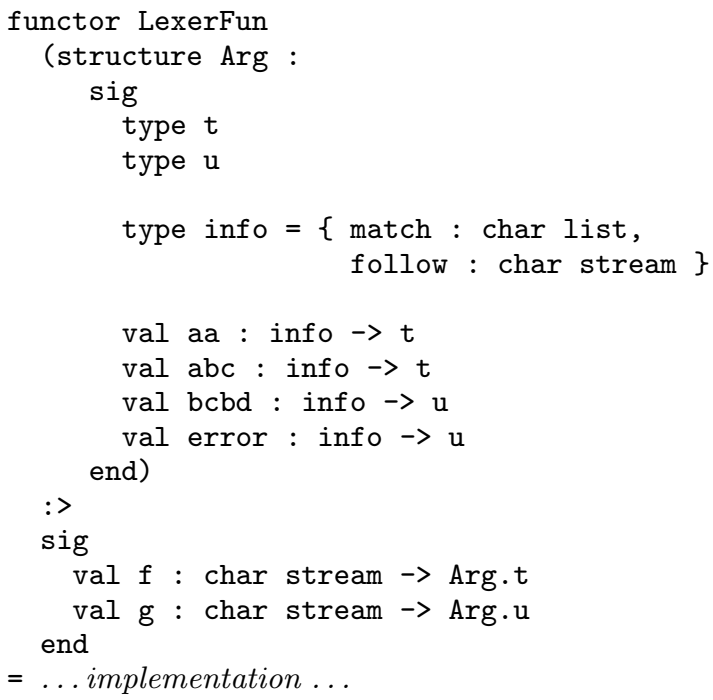

\section{Recursion in actions}

One important functionality for a lexer generator is the ability for actions to invoke the lexer recursively. For example,

\footnotetext{
${ }^{7}$ In contrast, the specification for $f$ was inexhaustive, so CM-Lex added a default error handler that raises an exception.
} 
it is common for a lexer, upon encountering whitespace, to skip the whitespace and call itself recursively (as in the example in Section 10 8

This can be problematic because it requires recursion between the lexer functor's argument and its result.

For example, consider a lexer that turns a stream of characters into a stream of words. The CM-Lex specification is:

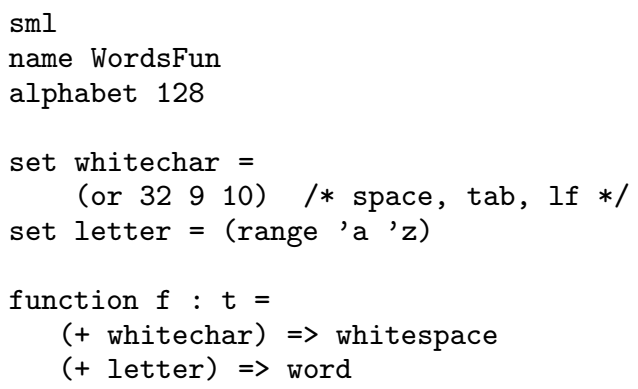

CM-Lex generates the functor:

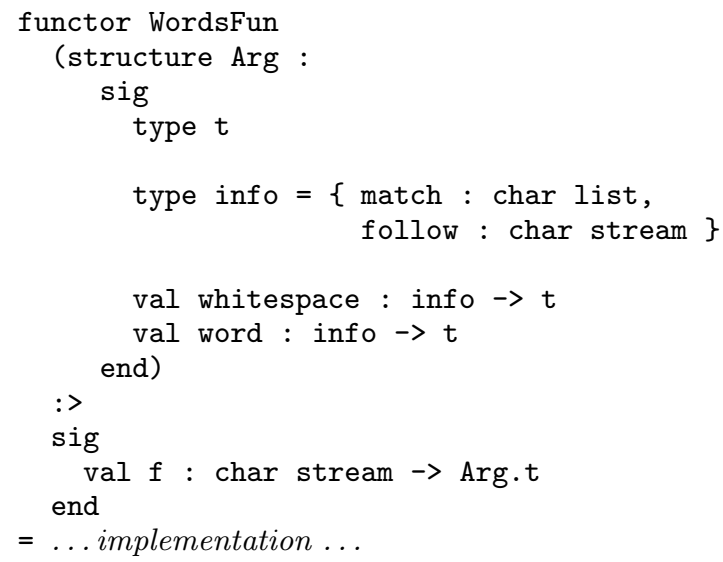

A natural way ${ }^{9}$ to implement the desired lexer would be with a recursive module definition:

${ }^{8}$ One way to accomplish this would be to structure the lexer with a driver loop (such as the function loop in Section 2], and for the action to signal the driver loop to discard the action's result and recurse. However, the earlier example notwithstanding, this is usually not the preferred way to structure a lexer.

${ }^{9}$ This simple implementation does not result in the best behavior from the lazy streams, because forcing the output stream causes the lexer to examine more of the input stream than is necessary to determine the output stream's first element. We illustrate a better way to manage laziness in Appendix $\mathrm{A}$ In any case, laziness is orthogonal to the issue being discussed here.

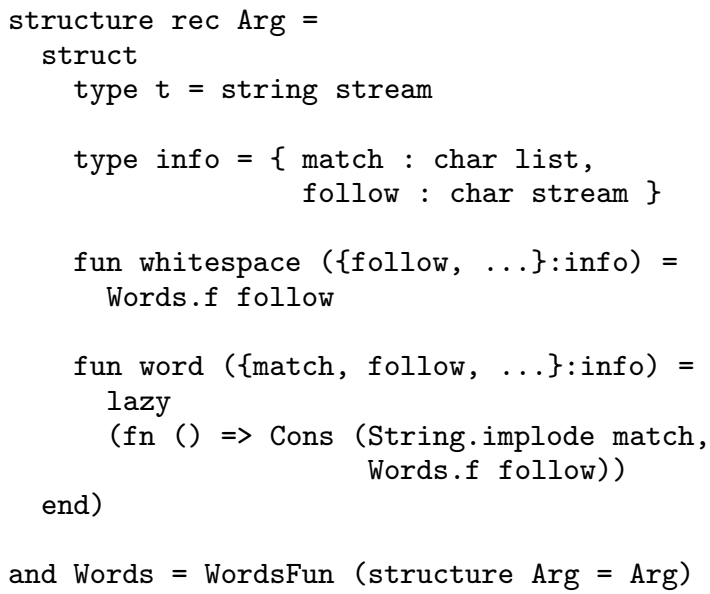

Unfortunately, recursive modules bring about a variety of thorny technical issues [2, 10, 4]. Although some dialects of ML support recursive modules, Standard ML does not.

As a workaround, CM-Lex provides recursive access to the lexer via a self field passed to each action. The info type is extended with a field self : self, where the type self is a record containing all of the lexing functions being defined. In this case:

type self $=\{f:$ char stream $\rightarrow t\}$

Using the self-augmented functor, we can implement the lexer as follows:

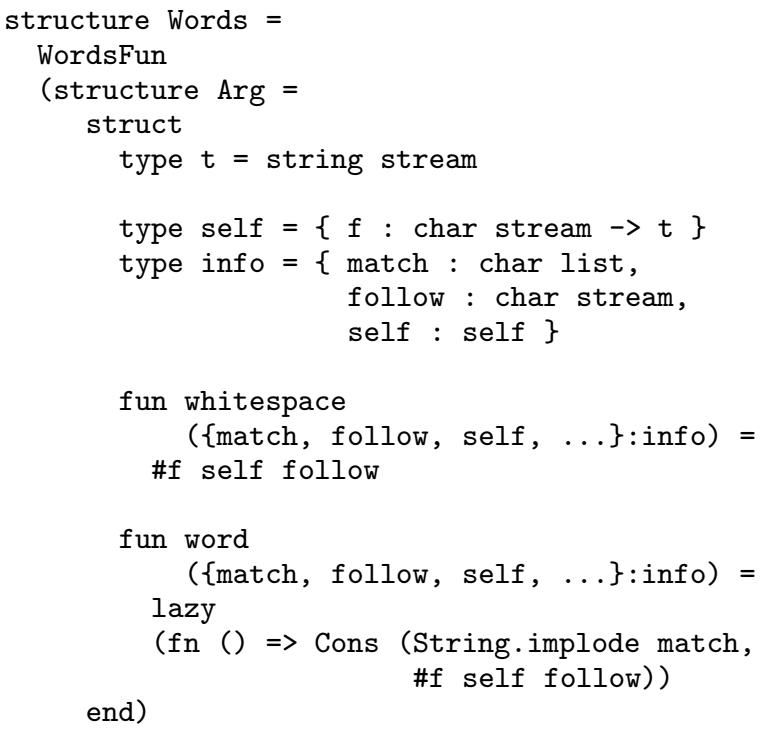

\section{Parsing Functor Generation}

The parser generator, CM-Yacc, works in a similar fashion to CM-Lex. A CM-Yacc specification for a simple arithmetic parser is: 
sml

name ArithParseFun

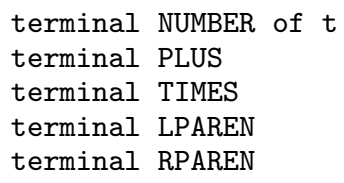

start Term

The specification says that the functor should be named ArithParseFun, and it declares five terminals, one of which carries a value of type $t$.

The specification then declares one nonterminal called Term, indicates that a term carries a value of type $t$, and gives four productions that produce terms 10 Numbers are attached to the symbols on the left-hand-side of a production that carry values that should be passed to the production's action. The number itself indicates the order in which values should be passed. Thus plus_term is passed a pair containing the first and third symbols' values.

The final line specifies that the start symbol is Term.

From this specification, CM-Yacc generates the following Standard ML code:

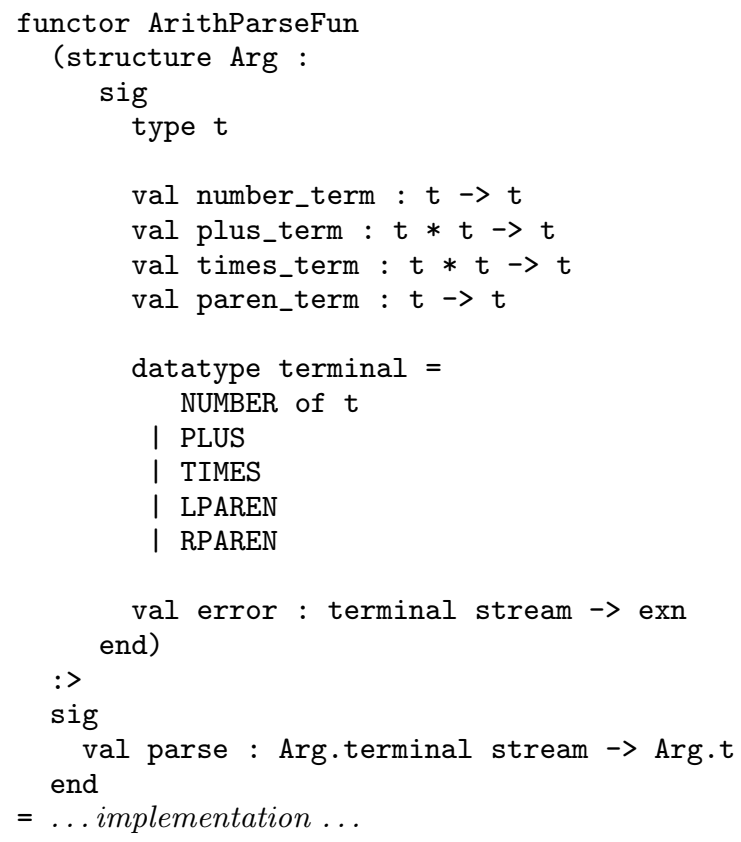

As before, the programmer supplies the type $t$ and the actions. (The actions need not be passed a self argument, because parser actions do not commonly need to reinvoke the parser.) He also supplies the terminal datatype and an error action, the latter of which takes the terminal stream

\footnotetext{
${ }^{10}$ This grammar is ambiguous, resulting in shift-reduce conflicts. The ambiguity can be resolved in either of the standard manners: by specifying operator precedences, or by refactoring the grammar.
}

at which a syntax error is detected and returns an exception for the parser to raise. For example:

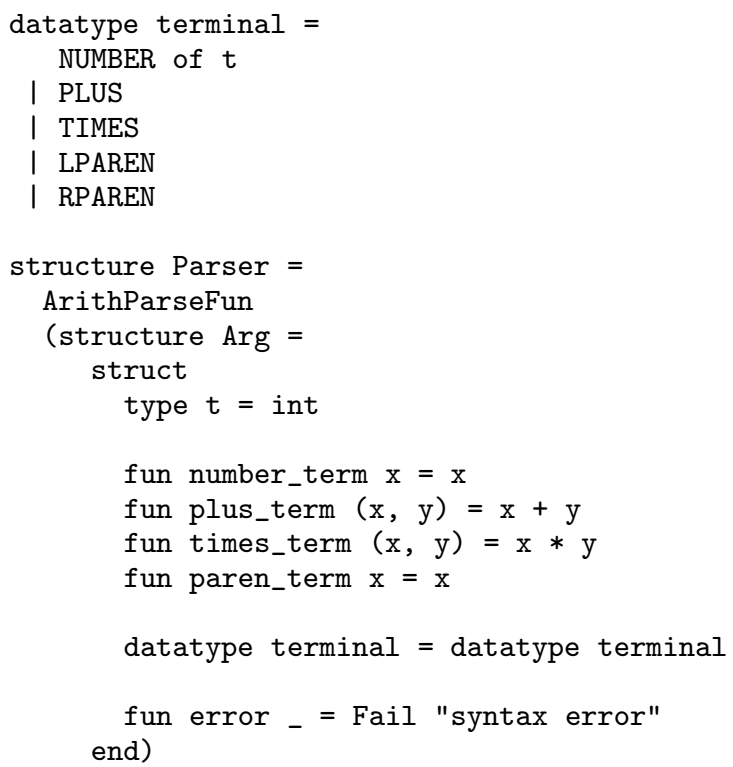

Then our parser is Parser.parse : terminal $\rightarrow$ int.

Note that we use datatype copying (a little-known feature of Standard ML) to copy the terminal datatype into the Arg structure. If the datatype were defined within the Arg structure, there would be no way to use it outside. OCaml does not support datatype copying, but one can get the same effect by including a module that contains the datatype.

\section{Functors in Haskell}

In broad strokes the Haskell versions of CM-Lex and CMYacc are similar to the ML versions. In one regard, they are simpler: In Haskell all definitions are mutually recursive, so no special functionality is required to allow lexer actions to reinvoke the lexer.

However, Haskell does not support functors, the central mechanism we exploit here. Instead, we built an ersatz functor from polymorphic functions.

Recall the CM-Lex specification from Section 2.1. reprised in Figure 2 From that specification, CM-Lex generates a module (in the Haskell sense) named LexerFun with the following exports:

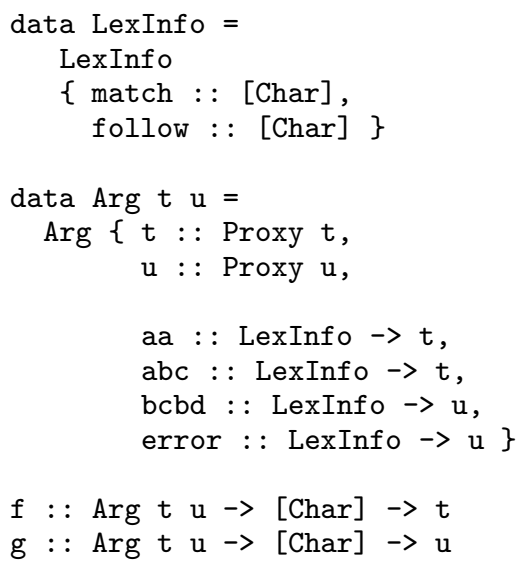




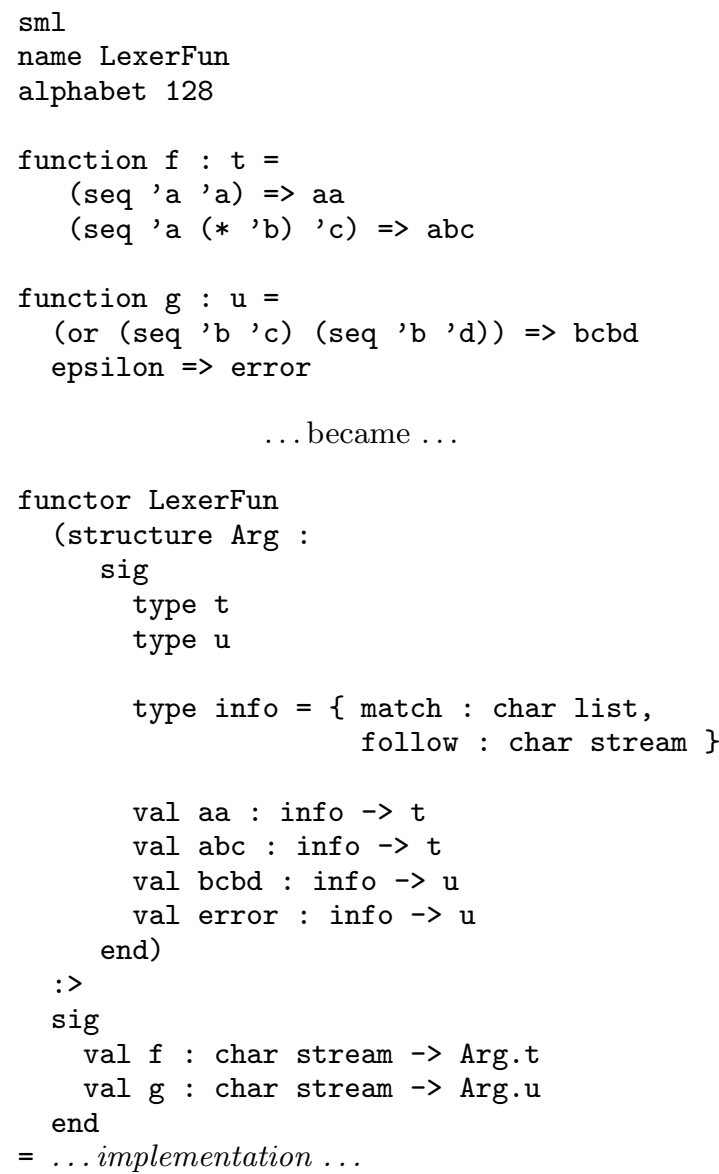

Figure 2: The example from Section 2.1

Compare this with the ML version, also reprised in Figure 2 The type Arg represents the argument to the functor. It contains implementations for the four actions aa, abc, bcbc, and error.

It also contains implementations for the two types $t$ and u. Haskell does not support type fields like an ML structure, but we can get a similar effect by including proxy fields with the types Proxy $t$ and Proxy $u$. The programmer then fills them in with the term Proxy : : Proxy $\mathrm{T}$ for some $\mathrm{T}$

Proxy [3] is a type constructor in the Haskell standard library that is designed for this sort of use. For any type constructor C, the type Proxy $\mathrm{C}$ has a single data constructor Proxy. The Proxy type constructor is poly-kinded, so C need not have kind $*$.

An alternative would be to leave out the type fields altogether and allow type inference to fill them automatically. We believe it would be a misstep to do so. The type implementations are critical documentation that should be given explicitly in the program. Moreover, leaving out the type implementations would bring back the possibility that the type checker would misattribute the source of a type error.

The functor's output is factored into two separate polymorphic functions that each take the functor argument as an argument. Since the type arguments $t$ and $u$ are propagated to the result types of the lexing functions, they must also appear as explicit parameters of the type Arg.

The Haskell version of CM-Yacc builds an ersatz functor in a similar fashion. However, while the ML version specified the terminal type as an input to the parser functor, there is no way to specify a datatype as an input to an ersatz functor. Instead, the parsing module defines the terminal datatype and passes it out.

In the example above, CM-Lex was used in purely functional mode. Consequently, the input stream was simply a character list, since Haskell lists are lazy already. Alternatively, CM-Lex and CM-Yacc can be directed to generate monadic code, which allows the lexer or parser to deal with side effects, either in the generation of the input stream (e.g., input read from a file) or in the actions. Doing so incurs some complications - it is important that the input stream be memoizing and not every monad is capable of supporting the proper sort of memoization 2 - but these complications are orthogonal to the functor mechanism discussed here and are beyond the scope of this paper.

\section{Conclusion}

We argue that functor generation is a cleaner mechanism for source-code-generating tools than assembling snippets of disembodied code. The resulting functor makes no demands on the surrounding code (other than a few standard libraries), and so it is guaranteed to type check 13 The programmer never need look at the generated code.

\footnotetext{
${ }^{11}$ Alternatively, one could give the proxy fields the bare types $t$ and $\mathrm{u}$ and fill them in with undefined : : $\mathrm{T}$, but that approach would be more awkward in the monadic case in which we also need to specify a monad. A monad has kind $* \rightarrow *$ and therefore does not have elements.

${ }^{12}$ Monads such as IO and ST that support references also support memoization, and Identity supports it trivially (since no memoization need ever be done), but most others do not.

${ }^{13}$ More precisely, it is guaranteed to type check in an initial context containing standard libraries and other module definitions. Unfortunately, Standard ML does not quite enjoy the weakening property, so the resulting functor is not guaranteed to type check in any context. Pollution of the namespace with datatype constructors and/or
} 
In contrast, with a snippet-assembling tool, an error in any snippet will - even in the best case - require the programmer to look at generated code containing the snippet. More commonly, the programmer will need to look at lots of generated code having nothing to do with the erroneous snippet.

We have demonstrated the technique for lexer and parser generation, but there do not seem to be any limitations that would preclude its use for any other application of sourcecode generation.

\section{A A Full Example}

As a more realistic example, we implement a calculator that processes an input stream and returns its value. For simplicity, the calculator stops at the first illegal character (which might be the end of the stream). The lexer specification is:

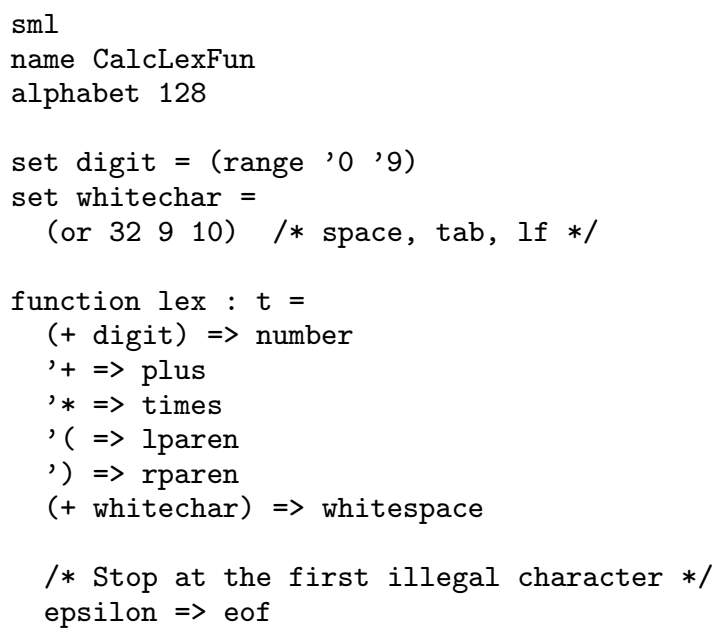

which generates:

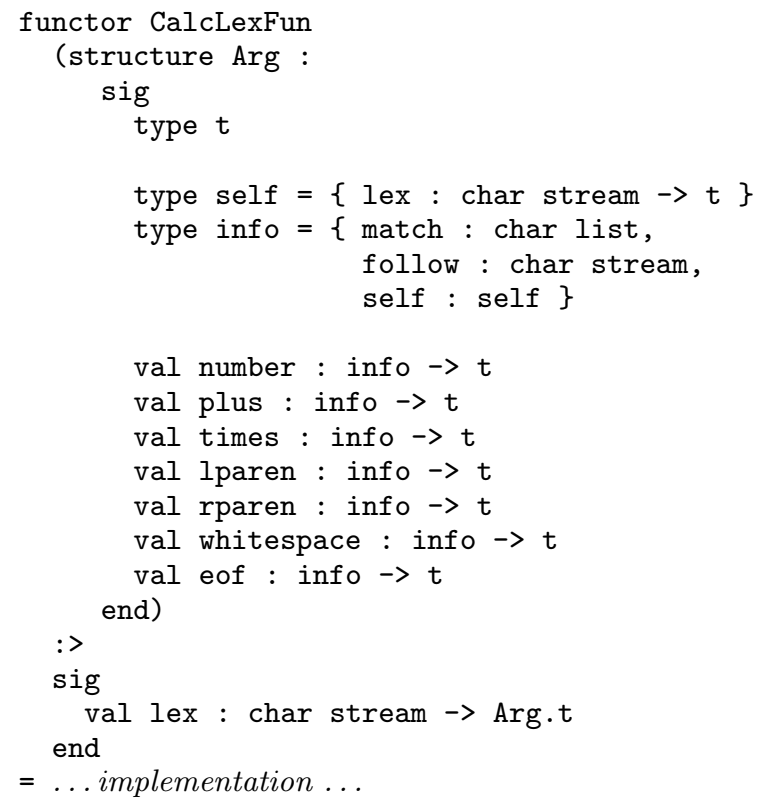

infix declarations for identifiers that are used within the generated functor will prevent it from parsing correctly. This is one reason why it is considered good practice in SML for all code to reside within modules. and the parser specification is:

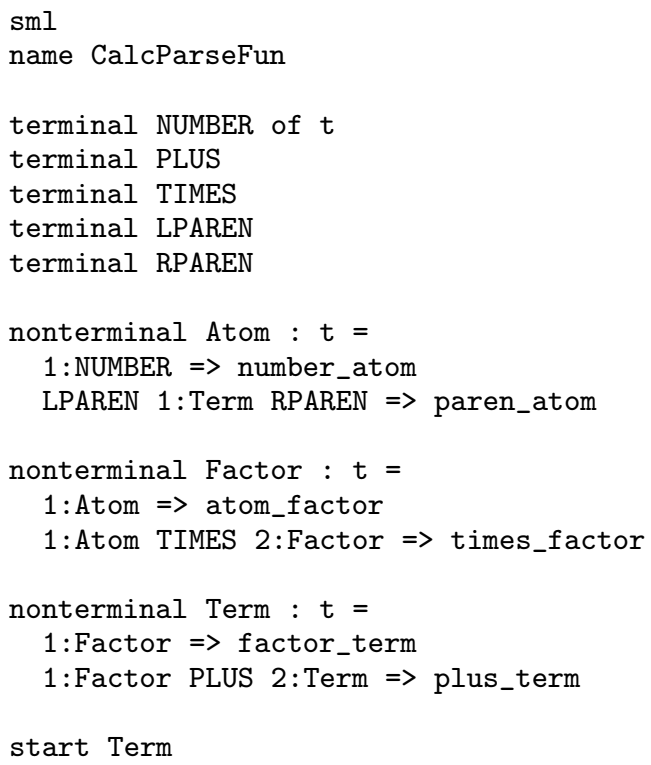

which generates:

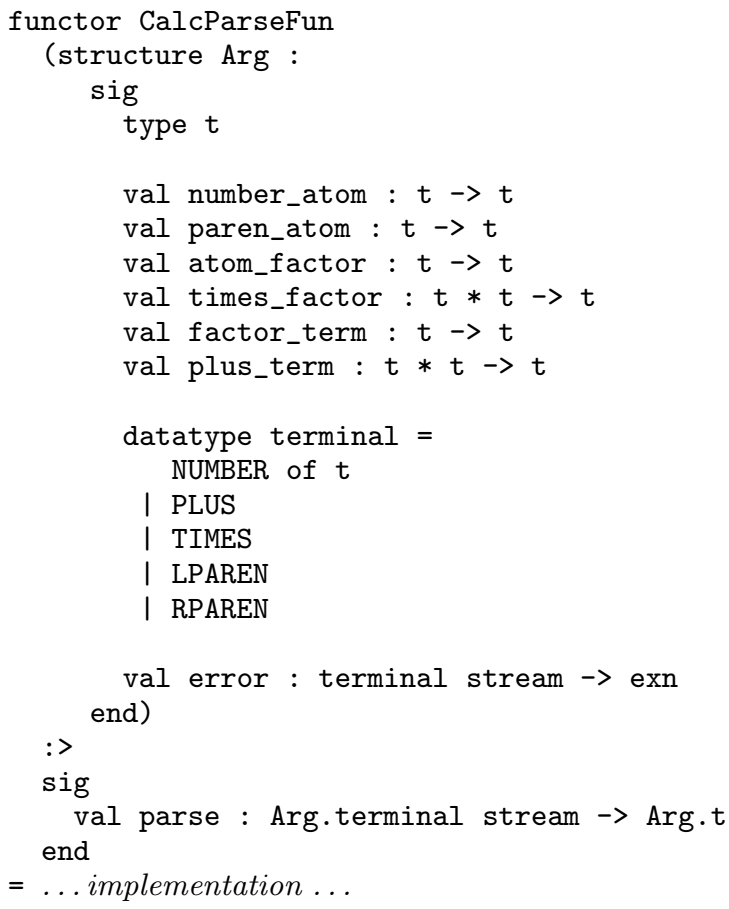

We then assemble the calculator as follows:

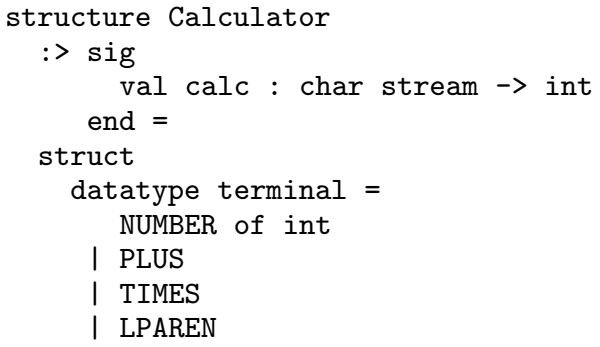




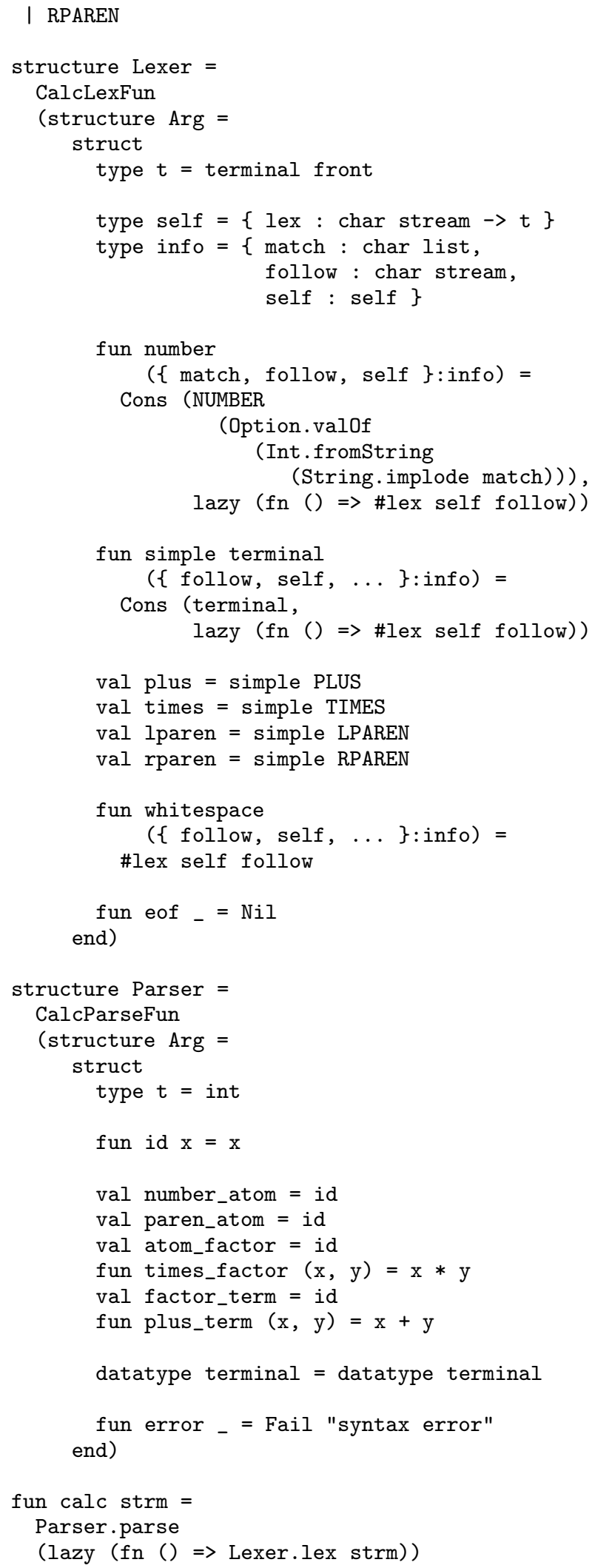

\section{References}

[1] Andrew W. Appel, James S. Mattson, and David R. Tarditi. A lexical analyzer generator for Standard $M L$, October 1994. Available at Www.smlnj.org/doc/ML-Lex/manual.html

[2] Karl Crary, Robert Harper, and Sidd Puri. What is a recursive module? In 1999 SIGPLAN Conference on Programming Language Design and Implementation, pages 50-63, Atlanta, May 1999.

[3] Data.Proxy. Online documentation at https://hackage.haskell.org/package/base-4.7.0.0/docs/Data2014. Retrieved October 21, 2017.

[4] Derek Dreyer. Understanding and Evolving the $M L$ Module System. PhD thesis, Carnegie Mellon University, School of Computer Science, Pittsburgh, Pennsylvania, May 2005.

[5] Richard Frost and John Launchbury. Constructing natural language interpreters in a lazy functional language. The Computer Journal, 32(2), 1989.

[6] Graham Hutton. Higher-order functions for parsing. Journal of Functional Programming, 2(3), 1992.

[7] Stephen C. Johnson. Yacc - yet another compiler compiler. Technical Report 32, Bell Laboratories Computing Science, Murray Hill, New Jersey, July 1975.

[8] Eugene Kohlbecker, Daniel P. Friedman, Matthias Felleisen, and Bruce Duba. Hygienic macro expansion. In 1986 ACM Conference on Lisp and Functional Programming, pages 161-161, 1986.

[9] M. E. Lesk. Lex - a lexical analyzer generator. Technical Report 39, Bell Laboratories Computing Science, Murray Hill, New Jersey, October 1975.

[10] Claudio V. Russo. Types for Modules. Number 60 in Electronic Notes in Theoretical Computer Science. Elsevier, January 2003.

[11] Olin Shivers. The SRE regular-expression notation, August 1998. Post to the comp.lang.scheme newsgroup, now archived at http://www.scsh.net/docu/post/sre.html

[12] Walid Taha and Tim Sheard. MetaML and multi-stage programming with explicit annotations. Theoretical Computer Science, 248(1-2), 2000.

[13] Aaron Turon and John Reppy. SML/NJ Language Processing Tools: User Guide, September 2015. Available at wWw.smlnj .org/doc/ml-lpt/manual.pdf 